\title{
Photonic Nanojet engineering: Focal point shaping with scattering phenomena of dielectric microspheres
}

\author{
Myun-Sik Kim*a ${ }^{\text {a }}$, Toralf Scharf ${ }^{\mathrm{a}}$, Stefan Mühlig ${ }^{\mathrm{b}}$, Carsten Rockstuhl ${ }^{\mathrm{b}}$, and Hans Peter Herzig ${ }^{\mathrm{a}}$ \\ ${ }^{a}$ Optics \& Photonics Technology Laboratory, Ecole Polytechnique Fédérale de Lausanne (EPFL), \\ Neuchâtel, CH-2000, Switzerland \\ ${ }^{\mathrm{b}}$ Institute of Condensed Matter Theory and Solid State Optics, Friedrich-Schiller-Universität Jena, \\ PF 07737 Jena, Germany
}

\begin{abstract}
We experimentally engineer Nanojets produced by dielectric spheres by varying the illumination and observe the effect with a high-resolution interference microscope (HRIM). Converging and diverging spherical wavefronts and BesselGauss beams are considered. We find that the diverging wavefront pushes Nanojets away from the surface of the sphere without change of the spot size. This allows earning several micrometers of working distance contrary to the Nanojet confined at the sphere's surface. When the radius of curvature of the incident wavefront is greater than about 5 times the sphere size, the Nanojet moves back to the sphere surface like it is found for plane wave incidence. On-axis BesselGauss beam illumination with the central lobe covering the whole sphere leads to the same results as the plane wave case. Off-axis Bessel beam illumination can generate multiple-spot Nanojets. We demonstrate the separation of such spots of about $220 \mathrm{~nm}$ at $642 \mathrm{~nm}$. This separation is smaller than the feature sizes defined by the diffraction limit at this wavelength. We discuss briefly applications of engineered Nanojets for nano-lithography and near-field sensing.
\end{abstract}

Keywords: Photonic Nanojet, beam shaping, wavefront measurement, interference microscope, nano-lithography

\section{INTRODUCTION}

Light scattering phenomena from small particles are well approximated by Mie theory for sizes of the particle greater than the wavelength ${ }^{1-2}$. Especially when these scattering objects are micro-size dielectric spheres, high-intensity, narrow beam-width, and elongated spots are generated on the shadow-side surface of the sphere. The phenomenon is known as photonic Nanojet ${ }^{3-4}$. Numerous simulation and experimental studies have been reported concentrating on a plane wave illumination. There are a few studies which apply focused beam to reduce spot sizes of a photonic Nanojet ${ }^{5}$. One of the main streams have been focusing on the key parameters depending on the working conditions, such as the refractive index of the sphere and background medium, the size of the sphere and the working wavelength. The others are application studies for sensing and metrology, optical trapping, nano-patterning, and optical data storage. Among a few experimental approaches, the direct experimental observation of Nanojets is reported only once ${ }^{6}$. Contrary to the freedom in simulations, tuning the working conditions is complex and sometimes not feasible in the experiment. In addition, the high-resolution measurement systems, such as confocal microscopy or scanning near-field microscopy (SNOM), are required to resolve small Nanojet spots. Varying the illumination conditions is difficult in those systems where illumination is usually defined by particular conditions.

In our study we experimentally engineer Nanojets by varying the illumination and investigate the modified properties of engineered Nanojets with a high-resolution interference microscope (HRIM). The HRIM system allows us to manipulate the incident illumination and to measure the wavefronts of incident and emerging beams in situ. We start with a plane wave illumination for reference, and then compare the properties of Nanojets generated by different illumination conditions. For a plane wave illumination, we present not only the experimental results but also the corresponding simulations. With a good agreement with simulations, we trust our experimental setup to work within theoretical framework. Since modifying the illumination conditions is usually more difficult in simulations, we investigate the engineering of the photonic Nanojet without corresponding simulations. Starting point is the wavefront of the incident beam. Converging and diverging spherical wavefronts are generated by defocusing from the entrance plane of the sphere (the bottom of the sphere). A focused Bessel beam (Bessel-Gauss beam) is considered as an alternative of a plane wavefront which shows localized and discrete plane wavefronts within the propagating length.

*myunsik.kim@epfl.ch; phone 41-32-718-3279; fax 41-32-718-3201; http://opt.epfl.ch/ 


\section{EXPERIMENTAL CONDITIONS}

\subsection{High-resolution interference microscopy setup details}

High-resolution interference microscopy is already proven as a powerful tool for characterizations of micro-size optical elements, such as microlenses and gratings ${ }^{7-8}$. HRIM is working in transmission by employing a Mach-Zehnder type interferometer as shown in Fig. 1(a). Three single mode polarized laser diodes with different powers and wavelengths are used to investigate the wavelength dependence in visible spectrum (CrystaLaser, 642nm: DL640-050-3, 532nm: IRGCL-025-S, 405nm: BCL-040-405-S). A polarizing beam splitter (PBS) divides intensities to be sent in a reference and an object arm with adjustable energy ratio. Half wave plates (HWP) and Glan-Taylor (G-T) polarizers are used to adjust the intensities and to optimize the contrast of the interference fringes. In the object arm, an expanded collimated beam (a plane wave) illuminates the system under investigation, which is mounted on a precision piezo stage with a z-scan range of $500 \mu \mathrm{m}$ and a nominal accuracy of $1 \mathrm{~nm}$ (MAD LAB CITY, NANO Z500). This z-axis piezo stage allows us to precisely find the plane of interest at the highest resolution. In the reference arm, a piezo-electrically driven mirror is mounted to change the optical path lengths. The phase distribution of the wave field is obtained by measuring the interference fringes at different mirror positions and employing a classical 5-frame algorithm ${ }^{9}$. In this scheme, 5 frames of the intensity pattern are recorded, each frame being shifted in phase by adding an additional phase of $\lambda / 4$. This interferometric function allows measuring and monitoring the wavefronts of the incident illumination beams during the engineering of the photonic Nanojet.

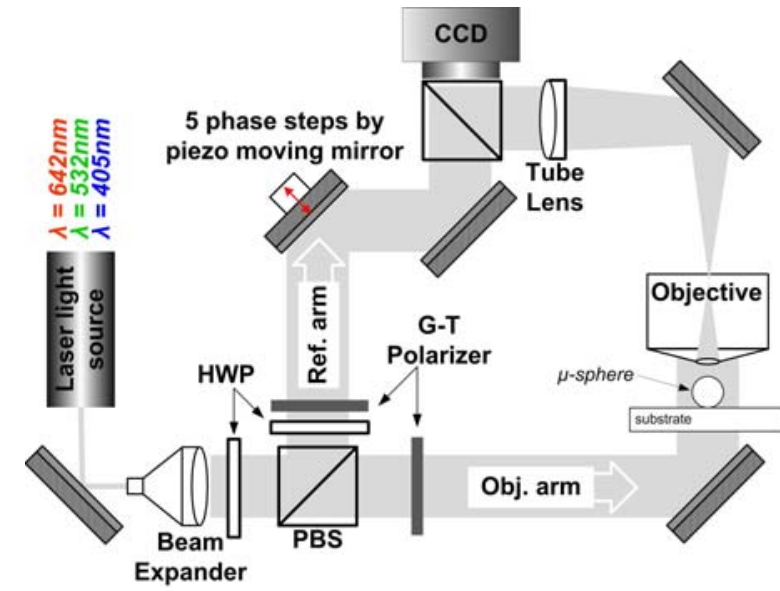

(a)

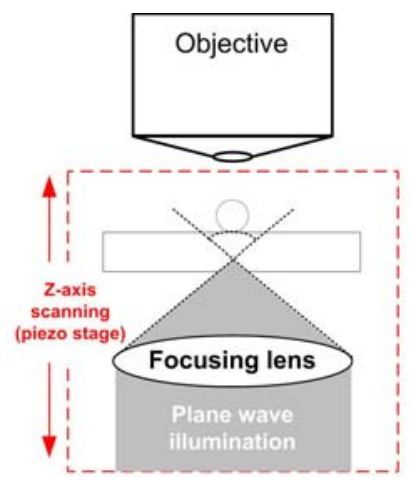

(b)

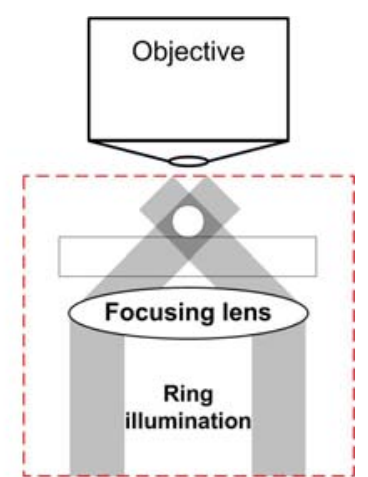

(c)

Figure 1. Schematic of the experimental setup and different illumination schemes. (a) System overview with a plane wave illumination. In the reference arm the piezo driven mirror modulates the optical path with 5 steps of $\lambda / 4$. (b) A spherical wave illumination and (c) a Bessel-Gauss beam illumination. Additional illumination elements are fixed on the same piezo stage to be moved together with a sample to keep illumination conditions constant.

In general, a high numerical aperture (NA) of the observation objective ensures high-resolution of the amplitude and phase measurements. Moreover, high magnification provides more pixels on an image sensor for small fields. We use a high NA objective, such as a 100X / NA 0.9 objective (Leica Microsystems, HC PL FLUOTAR). The resolution of an optical microscope is usually defined as the minimum resolvable distance between two points based on the Rayleigh criterion $^{10}$. The lateral resolution for this objective is $\Delta x=435 \mathrm{~nm}$, which is calculated with the formula $\Delta x=0.61 \lambda / N A$ with a wavelength of $642 \mathrm{~nm}$. Along the optical axis, the Rayleigh criterion can be applied with the simplified formula derived as $\Delta \mathrm{z}=\lambda \cdot \mathrm{n} /\left(\mathrm{NA}^{2}\right)$, where $\mathrm{n}$ is the refractive index of medium ${ }^{11}$. The calculated axial resolution at $642 \mathrm{~nm}$ wavelength is $\Delta z=793 \mathrm{~nm}$. At $100 \mathrm{X}$ magnification, a pixel on a charge-coupled device (CCD) sensor (Sicon Corporation, CFW1312M camera with SONY ICX205AK image sensor of 1360 x 1024 pixels) corresponds to $46.5 \mathrm{~nm}$ in the object plane. This leads to the maximum field of view of the CCD camera of $64 \mathrm{x} 48 \mu \mathrm{m}^{2}$, which is sufficiently large to image the incident wavefronts and Nanojet microspheres. 


\subsection{Illumination conditions}

An expanded collimated beam (a plane wave) illuminates the sample as shown in Fig. 1(a). We can additionally insert illumination elements in the optical path underneath the sample as depicted in Figs. 1(b) and 1(c), such as a focusing lens for spherical wave illumination and ring illumination to create Bessel-Gauss beams. These illumination elements are fixed on the same piezo-stage as the sample. Since we assure a plane wave incidence onto these elements, the sample together with illumination elements can be scanned in the axial direction without changing the illumination conditions. It leads to three-dimensional data of amplitude and phase. Figure 2 presents examples for different illumination models without sample, hence only the illumination spot is shown. Figure 2(a) is the interference fringe image of a propagating plane wave in the axial direction, which is measured by the optical path modulation technique in the interferometer. Tow typical focused Gaussian beams for a low NA (0.15) and a high NA (0.9) are shown in Figs. 2(b) and 2(c) with their intensities, respectively. Defocusing them provides converging and diverging spherical wavefront illuminations. When we combine a focusing lens with a ring illumination, focused Bessel beams (Bessel-Gauss beams) are generated. The generated beam is not a perfect Bessel beam as could be obtained by using an axicon or micro-size-opening ring apertures. Results are shown in Figs. 2(d) and 2(e) and indicate the much longer focal depths of such beams usually called the propagation lengths. The phase measurements allow us to monitor and control the incident wavefront during the experiments. The wavefronts of the Bessel beams are plane within the propagation length. Within the propagation length of the Bessel beam, discrete and localized plane wavefronts are found beside the main intensity lobe. These side lobs will be applied to engineer the photonic Nanojets later in the section 4.

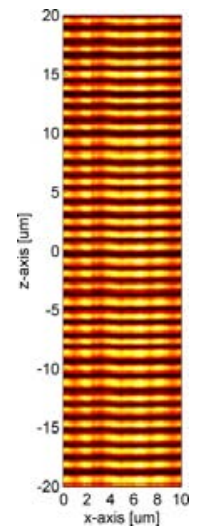

(a)

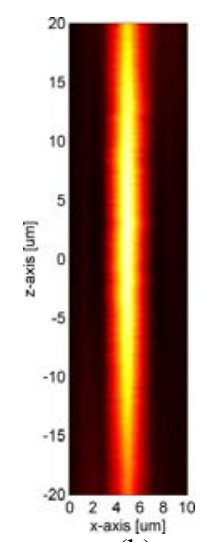

(b)

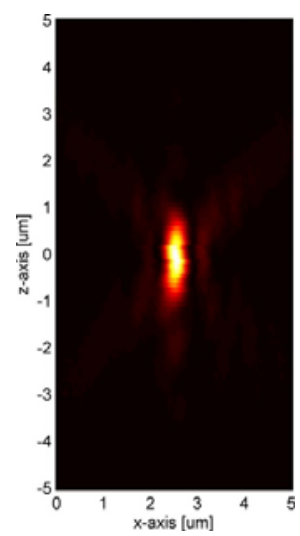

(c)

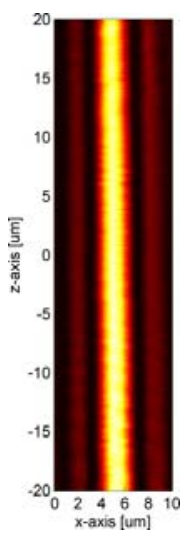

(d)

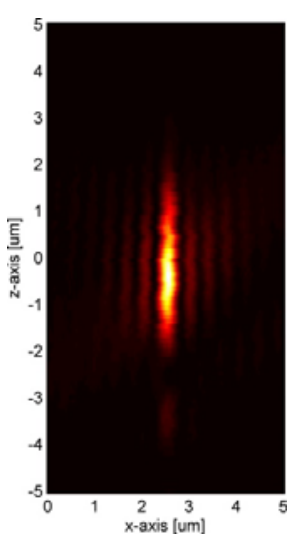

(e)

Figure 2. The $x-z$ slices of the measured 3D intensity data of various illuminations, which were measured without a sample in HRIM: (a) a plane wave, (b) low NA, (c) high NA focused Gaussian beams, (d) low NA and (e) high NA Bessel-Gauss beams. Scales are indicated in the corresponding axes. Intensity is attenuated in order to avoid saturation on a CCD sensor.

\section{PHOTONIC NANOJET BY A PLANE WAVE ILLUMINATION}

We start from the standard photonic Nanojets arrangement and plane wave illumination for reference. We verify the results and characteristics of the system by comparing measurement results with simulations. In our experiments we deposit borosilicate glass microspheres of different size (Duke Scientific, $\mathrm{n}=1.56$ at $589 \mathrm{~nm}$ ) on the glass substrate. The measured 3D intensity distributions of the photonic Nanojets from a 2- $\mu \mathrm{m}$ glass sphere with three different wavelengths and the corresponding simulation are shown in Figs. 3 and 4. Figure 3 shows the measured intensity distributions of Nanojets in the $x-z$ and the $x-y$ planes. The wavelength dependence has been explored by applying three different wavelengths available in our instrument. Since it is not a resonant phenomenon, we observe that the spot size has a direct relation to the working wavelength as it could be found in the focal spot of a conventional lens. Measurements are subjected to the diffraction limit of the observing objective. Figure 4 shows simulations of the same situation. Scattered fields are rigorously calculated by the Mie theory propagated in the far field. The angular spectrum is adapted to consider the finite numerical aperture of our observation objective. Back propagation leads to a reconstruction of the 3D amplitude and phase fields at the sphere position taken into account our observation conditions. 
The simulation results show a very good agreement with measurements as shown in Fig. 4. We see that our experimental system works within the theoretical framework and limits and that its performance is reliable with careful considerations of the diffraction limit. The longitudinal (axial) spot sizes corresponds also to a diffraction limited imaging, but the position of the plane of maximum intensity is found very close to the correct position because diffraction limits not the position accuracy but the measurable size of an object. As there is no evident resonance behavior, we will work with a single wavelength $(642 \mathrm{~nm})$ to investigate feasibility of the Nanojet engineering.

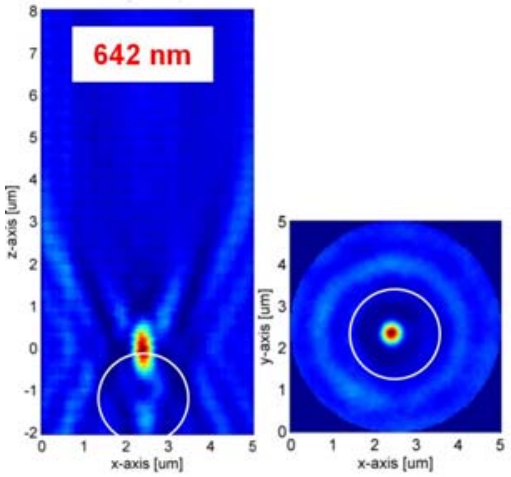

(a)

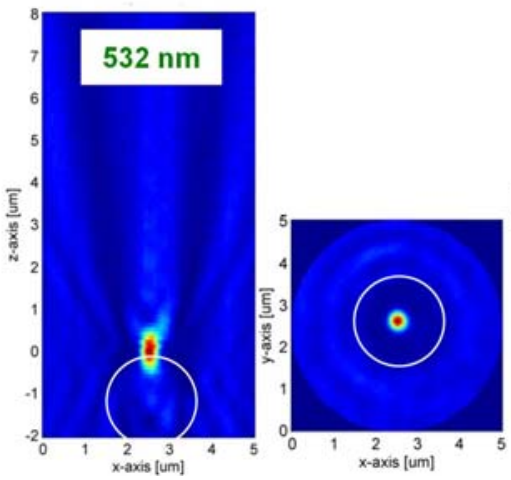

(b)

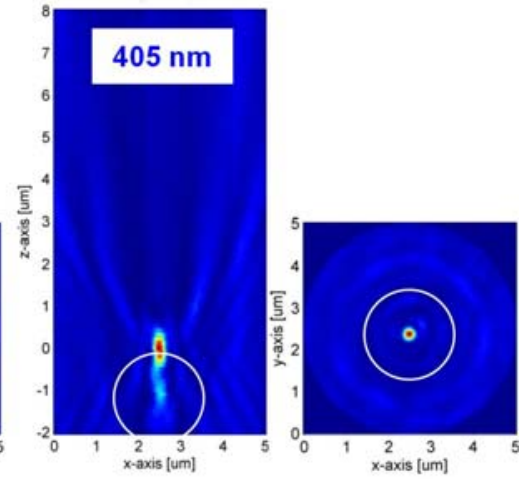

(c)

Figure 3. Measured intensity distributions of photonic Nanojets from a 2- $\mu \mathrm{m}$ glass sphere for three wavelengths: (a) 642 $\mathrm{nm}$, (b) $532 \mathrm{~nm}$ and (c) $405 \mathrm{~nm}$. The top of the sphere is set to the $\mathrm{z}=0 \mu \mathrm{m}$. The white circle indicates a $2-\mu \mathrm{m}$ sphere.

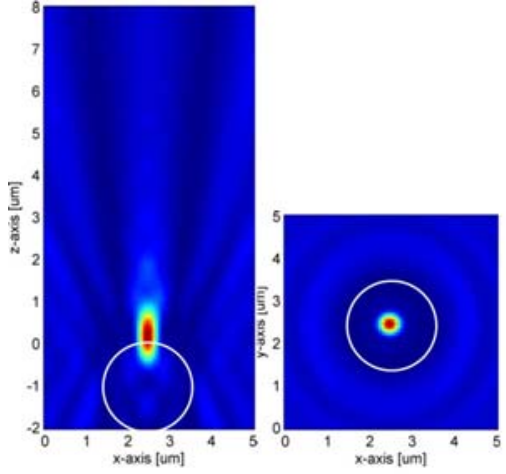

(a)

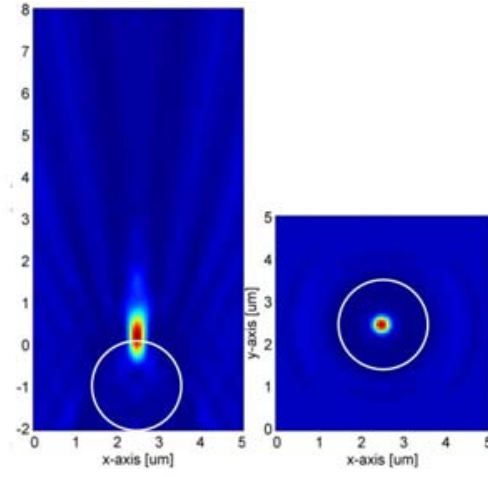

(b)
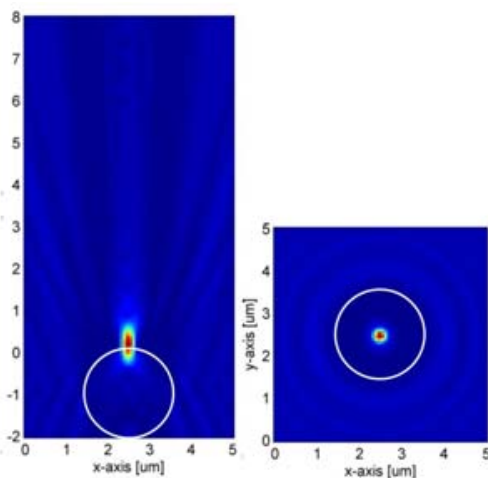

(c)

Figure 4. Corresponding simulations to Fig. 3: (a) $642 \mathrm{~nm}$, (b) $532 \mathrm{~nm}$ and (c) $405 \mathrm{~nm}$. They are rigorously calculated by the Mie theory and the scalar propagation technique (with the consideration of the NA of the objective). They show a good agreement with the measured data in Fig. 3.

\section{PHOTONIC NANOJET ENGINEERING}

As demonstrated in section 2.2, we can experimentally manipulate the kind of wavefront of incident beams at the entrance plane of the sphere. First, we consider a spherical wavefront, which is generated by defocusing. Second, a localized plane wavefront found in Bessel-Gauss beams will be examined and compared to plane wave illumination. For experimental convenience a microspheres with larger diameter, in our case with a diameter of $d=12 \mu \mathrm{m}$, is investigated. The observation wavelength is fixed at $642 \mathrm{~nm}$ wavelength. Results obtained with a 2- $\mu \mathrm{m}$ microsphere will be briefly discussed and compared. 


\subsection{Converging and diverging spherical wavefronts illumination}

We recall the situation of focusing for a conventional lens: For a lens we find its focal point moving backward and forward from the principal focal plane found for plane wave illumination and depending on the curvature of the incident wavefront. We apply this simple mechanism to adjust the focal plane (plane of maximum intensity) of the photonic Nanojet. Note that the photonic Nanojet is not a refraction phenomenon, which is the working principle of a lens, but a scattering effect. Therefore the phenomena could not be explained with straightforward arguments that apply for the lens case. To generate spherical wave illuminations with wavefronts of different curvatures, we use a 50X / NA0.9 objective (Leica Microsystems, HXC PL APO). This objective has no coverslip correction. Because of the substrates thickness of $170 \mu \mathrm{m}$ spherical aberrations in the illumination path are found. This unwanted aberration shows interesting consequence, such as interference between engineered Nanojets and fields from marginal rays, which are escaped from the geometrical shadow of the sphere. It leads to multiple focal points as shown in the $\mathrm{x}-\mathrm{z}$ intensity distribution of Fig. 5(c). Such effects will not be discussed further here. In what follows we focus on the engineering of the focal length.
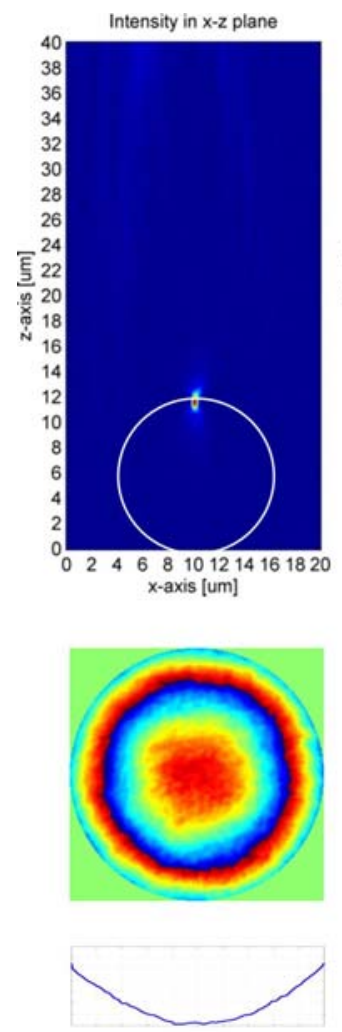

(a)
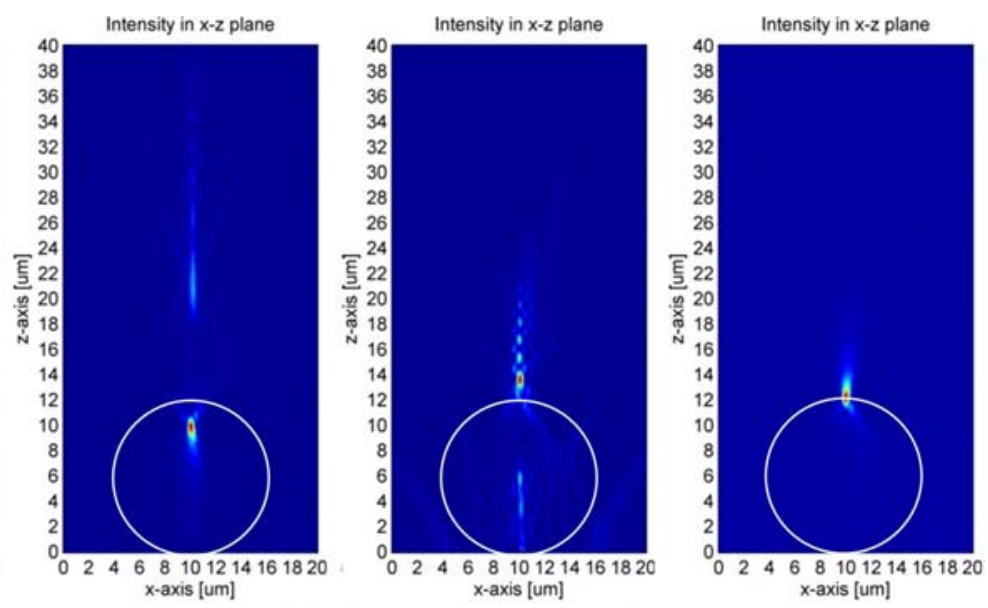

Measured 2D phase at the entrance plane

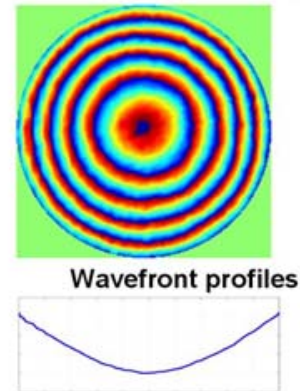

(b)
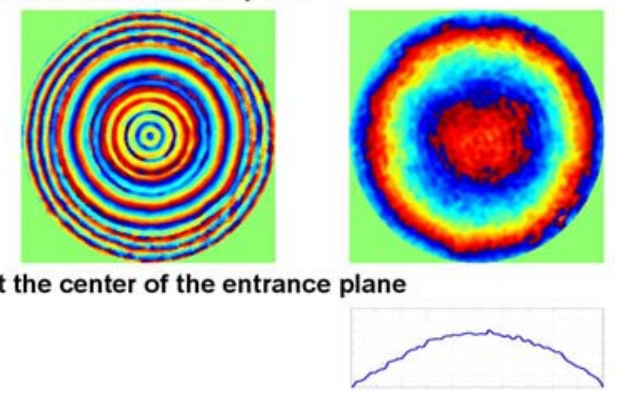

(c) (d)

Figure 5. Measured intensity of the engineered photonic Nanojets by converging and diverging spherical wave illuminations: (a) converging wavefront with a ROC of $50 \mu \mathrm{m}$, (b) converging wavefront with a ROC of $15 \mu \mathrm{m}$, (c) diverging wavefront with a ROC of $15 \mu \mathrm{m}$ and (d) diverging wavefornt with a ROC of $50 \mu \mathrm{m}$. Underneath of the $x-z$ intensity maps, the measured $x-y$ phase data and wavefront profiles at the plane of $z=0 \mu \mathrm{m}$ are shown. Although the wavefront profile of diverging wave with a ROC of $15 \mu \mathrm{m}$ was not obtained because of the spherical aberration, the ROC corresponds to the defocusing length, which is $15 \mu \mathrm{m}$ in (c). The white circle in the intensity images indicates a $12-\mu \mathrm{m}$ sphere.

In HRIM, we can precisely control the radius of curvature (ROC) of the incident wavefronts by measuring and monitoring the phase of the incident beams as shown in the phase maps and wavefront profiles of Fig. 5. As we expected, spherical wavefronts of different curvatures shift the focal plane of the Nanojet in the same manner as for a lens. Specifically, diverging spherical wavefronts pushes the Nanojet spot away from its principal focal plane (the shadow- 
side surface of the sphere) towards the far field as shown in Fig. 5(c). With this mechanism we can adjust the working distance of Nanojets within several microns. Moreover, when the ROC of the incident wavefront becomes greater than about 5 times the diameter of the sphere, the focal plane of the Nanojet comes back to the surface of the sphere. A 2- $\mu \mathrm{m}$ sphere shows the same behavior. We can conclude that a spherical wavefront, whose ROC is relatively greater than the object size, interacts with scattering object as a plane wave does. This gives us freedoms in the experiments because the high quality plane wave costs time and efforts.

\subsection{On-axis Bessel-Gauss beams: localized plane wavefronts}

A Bessel beam is a non-diverging propagating wave, whose wavefront is plane within its propagation length. Wavefronts of the Bessel beam is not an infinite plane wave but a discrete and localized plane wave within a small experimental volume. The size of the central lobe of the Bessel-Gauss beam depends on the numerical aperture of the focusing objective and it is slightly smaller than the focal spot found for Gaussian beams at a given numerical aperture. In order to illuminate full aperture of the sphere with the central lobe of a Bessel-Gauss beam, we use the practically lowest NA (0.15) focusing objective, which leads to a diameter of the central lobe of about $5 \mu \mathrm{m}$. A $2-\mu \mathrm{m}$ sphere is inserted in the optical path of the central lobe as shown in Fig. 6(b). Since the plane wavefronts illuminates the sphere, this finally leads to the same results as an expanded collimated beam (a plane wave) illumination. The spot size stays the same and the intensity maximum position is found on the surface of the sphere as shown in Fig. 6(c).

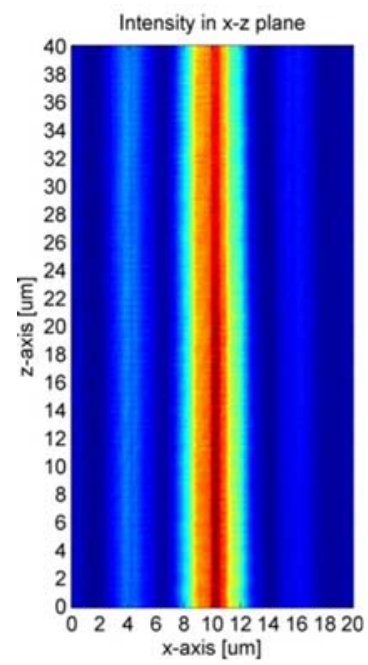

(a)

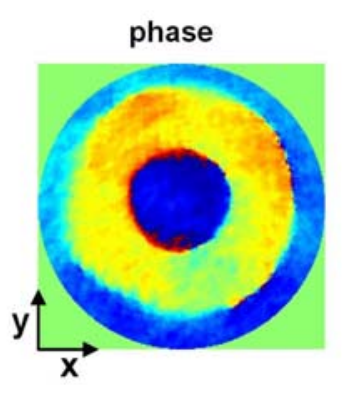

(b)

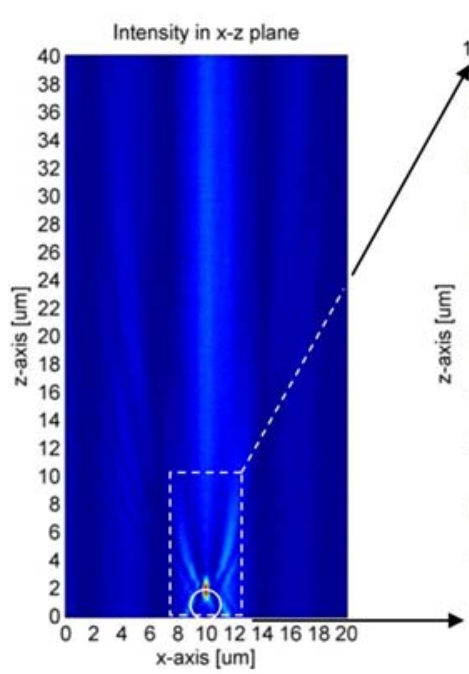

(c)

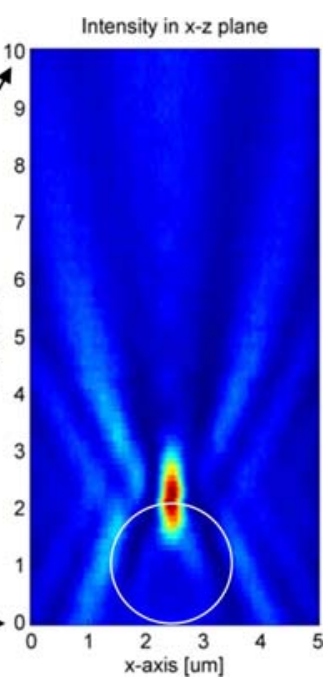

(d)

Figure 6. (a) The $x-z$ intensity distribution and (b) the $x-y$ phase map of the Bessel-Gauss beam without a sphere. (c) The Nanojet generated by on-axis Bessel beam illumination and (d) close-up image of the Nanojet near the sphere. The white circle indicates a $2-\mu \mathrm{m}$ sphere.

\subsection{Off-axis Bessel-Gauss beams: localized and discrete plane wavefronts}

An interesting phenomenon can be found when illumination the sphere with side lobs of the Bessel beam. We illuminate a 4- $\mu \mathrm{m}$ sphere with the off-axis Bessel-Gauss beam under the same experimental conditions as shown in Figs. 6(a) and 6(b), by placing the sphere off axis between two side lobes. The intensity zero rings separate the lobes and actually the wavefronts contributing to propagation of the whole beam. The result of this experiment is a two-spot Nanojet as shown in Figs. 7(a) and 7(b). The longitudinal slices of the measured 3D intensity data are shown in Figs. 7(c) - 7(e). The separation of two spots shows $220 \mathrm{~nm}$ at FWHM, which is about $40 \%$ smaller than the measured Nanojet spot size at a wavelength of $642 \mathrm{~nm}$. These phenomena are observed with spheres of different sizes. Such two-spot Nanojets can be applied to direct-write lithography at shorter wavelengths, such as a $405 \mathrm{~nm}$ or UV light. The particular intensity distribution allows creating structures with line widths that are much smaller than the diffraction limit. 


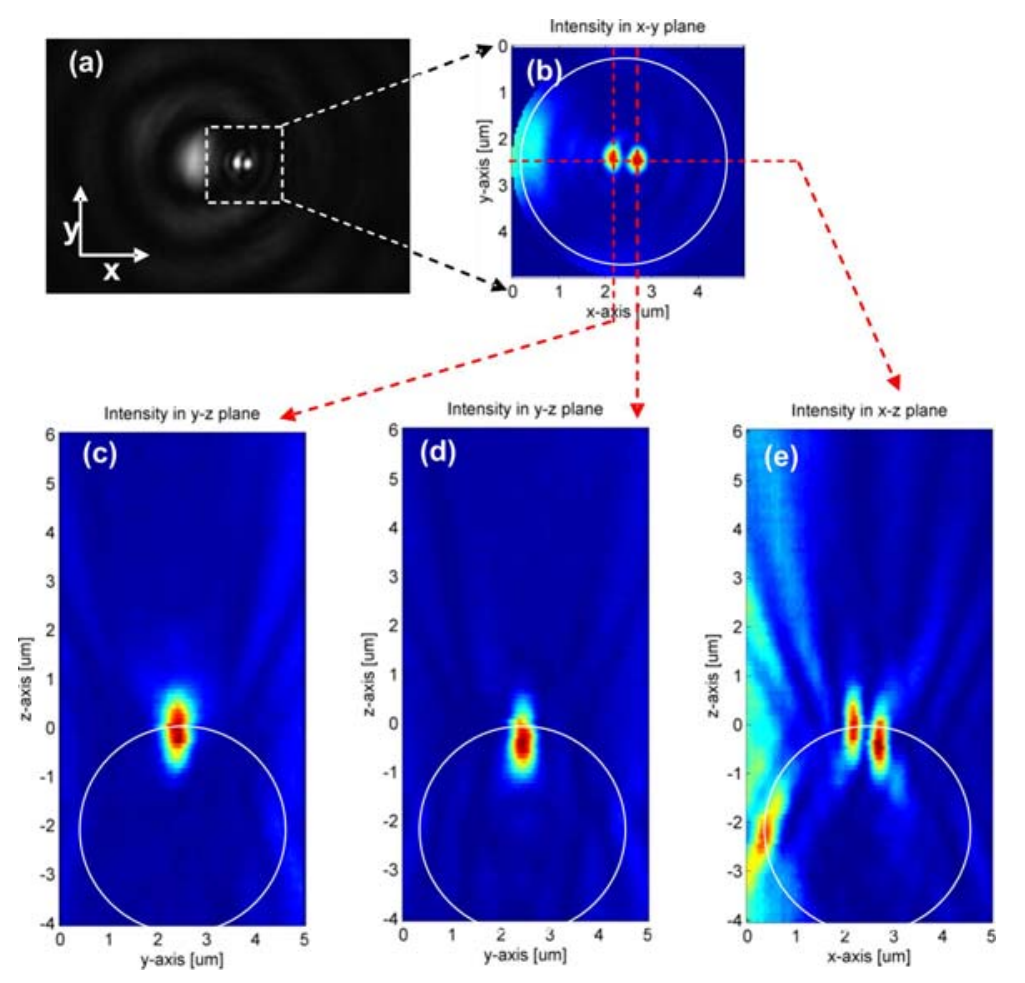

Figure 7. Measured intensity data of the two-spot Nanojet from a 4- $\mu \mathrm{m}$ sphere by off-axis Bessel-Gauss beam illumination: (a) a CCD image of the off-axis Bessel beam and two-spot Nanojet, (b) the x-y slice of 3D data at the top of the sphere (c) the $y-z$ slice at the center of the left spot, (d) the y-z slice at the center of the right spot and (e) the $x-z$ slice of the two spots. The white circle indicates a $4-\mu \mathrm{m}$ sphere.

\section{CONCLUSIONS}

We experimentally and theoretically studied the photonic Nanojets in a plane wave illumination with three wavelengths (red at $642 \mathrm{~nm}$, green at $532 \mathrm{~nm}$ and blue at $405 \mathrm{~nm}$ ) for reference. Simulations show a good agreement with experiments which guarantees the performance of our instrument, the HRIM. The wavelength dependence shows the conventional scaling-down effect that is found in imaging when no resonance effects are expected.

We investigated three different illumination conditions to engineer Nanojet spots. First, a spherical wavefront illumination has been studied. Spherical wavefronts show the ability to adjust the focal plane of the Nanojet depending on the curvature of the wavefront. When the Nanojet has the intensity maximum plane on the surface of the sphere, it is hard to utilize in practice because one needs to approach to the near field of the sphere. Especially, the diverging spherical wavefronts push the focal plane several micrometers away from the surface of the sphere. Engineered Nanojet provides a reasonable working distance for applications with a small working distance such as near-field sensing, wafer inspections or scanning microscopy. This works only in certain limits. If the radius of curvature of the wavefront is sufficiently larger than the size of the sphere, the Nanojet behaves like under plane wave illumination.

Second, Bessel-Gauss beam illumination has been considered as an alternative plane wavefront but confined illumination. Wavefronts of the Bessel beam are plane and the only difference is that they are discrete rather than continuous within a small experimental volume. In on-axis Bessel beam illumination and when the central intensity lobe covers the full aperture of the sphere gives results similar to plane wave illumination as excepted. Placing a sphere between two side lobes of the Bessel beam (off-axis Bessel beam) illumination generated a two-spot Nanojet. The separation of those spots is about $220 \mathrm{~nm}$ at $642 \mathrm{~nm}$ incidence which is much smaller than the diffraction limited feature size at this wavelength. Such narrow "dark-field" like two-spot Nanojets can be applied to the direct-write nano-lithography. 


\section{REFERENCES}

[1] Mie, G., "Beiträge zur Optik trüber Medien, speziell kolloidaler Metallösungen," Ann. d. Physik, 25 (4), 377445 (1907).

[2] Van de Hulst, H. C., [Light Scattering by Samll Particles], Dover, New York, chap. 9 (1981).

[3] Chen, Z., Taflove, A. and Backman, V.,"Photonic Nanojet enhancement of backscattering of light by nanoparticles: a potential novel visible-light ultramicroscopy technique," Opt. Express 12, 1214-1220 (2004).

[4] Li, X., Chen, Z., Taflove, A. and Backman, V.,"Optical analysis of nanoparticles via enhanced backscattering facilitated by 3-D photonic Nanojets," Opt. Express 13, 526-533 (2005).

[5] Devilez, A., Bonod, N., Wenger, J., Gérard, D., Stout, B., Rigneault, H. and Popov, E.,"Three-dimensional subwavelength confinement of light with dielectric microspheres," Opt. Express 17, 2089-2094 (2009).

[6] Ferrand, P., Wenger, J., Devilez, A., Pianta, M., Stout, B., Bonod, N., Popov, E. and Rigneault, H., "Direct imaging of photonic Nanojets," Opt. Express 16, 6930-6940 (2008).

[7] Rockstuhl, C., Märki, I., Scharf, T., Salt, M., Herzig, H. P. and Dändiker, R., "High Resolution Interference Microscopy: A Tool for Probing Optical Waves in the Far-Field on a Nanometric Length Scale," Current Nanoscience 2, 337-350 (2006).

[8] Kim, M.-S., Scharf, T. and Herzig, H. P., "Small size microlenes characterization by Multiwavelength High Resolution Interference Microscopy," Opt. Express 18, 14319-14329 (2010).

[9] Schwider, J., Burow, R., Elssner, K.-E., Grzanna, J., Spolaczyk, R. and Merkel, K., "Digital wave-front measuring interferometry: some systematic error sources," Appl. Opt. 22, 3421-3432 (1983).

[10] Born, M. and Wolf, E., [Principles of Optics, 7th (expanded) edition], Cambridge University Press, Cambridge, Chap 7 and 9 (1999).

[11] Gross, H., [Handbook of Optical Systems, vol. 3] Wiley-VCH, Weinheim, p. 126 (2007). 